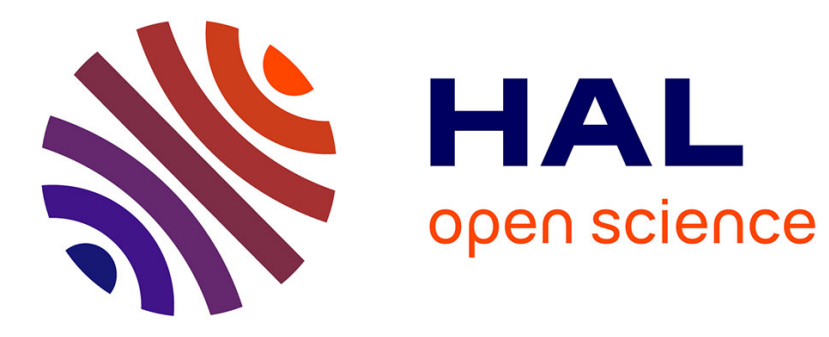

\title{
Quasi-rectilinear (MSK, GMSK, OQAM) co-channel interference mitigation by three inputs widely linear Fresh filtering
}

\author{
Pascal Chevalier, Rémi Chauvat, Jean-Pierre Delmas
}

\section{To cite this version:}

Pascal Chevalier, Rémi Chauvat, Jean-Pierre Delmas. Quasi-rectilinear (MSK, GMSK, OQAM) cochannel interference mitigation by three inputs widely linear Fresh filtering. ICASSP 2015: 40th International Conference on Acoustics, Speech and Signal Processing, Apr 2015, Brisbane, Australia. pp.2434 - 2438, 10.1109/ICASSP.2015.7178408 . hal-01272822

\section{HAL Id: hal-01272822 \\ https://hal.science/hal-01272822}

Submitted on 2 Dec 2020

HAL is a multi-disciplinary open access archive for the deposit and dissemination of scientific research documents, whether they are published or not. The documents may come from teaching and research institutions in France or abroad, or from public or private research centers.
L'archive ouverte pluridisciplinaire HAL, est destinée au dépôt et à la diffusion de documents scientifiques de niveau recherche, publiés ou non, émanant des établissements d'enseignement et de recherche français ou étrangers, des laboratoires publics ou privés. 


\title{
QUASI-RECTILINEAR (MSK, GMSK, OQAM) CO-CHANNEL INTERFERENCE MITIGATION BY THREE INPUTS WIDELY LINEAR FRESH FILTERING
}

\author{
Pascal Chevalier ${ }^{(1,2)}$, Rémi Chauvat ${ }^{(1,2)}$ and Jean-Pierre Delmas ${ }^{(3)}$ \\ (1) CNAM, CEDRIC laboratory, 75003 Paris, France \\ (2) Thales-Communications-Security, HTE/AMS/TCP, 92622 Gennevilliers, France \\ (3) Telecom SudParis, UMR CNRS 5157, 91011 Evry, France
}

\begin{abstract}
Widely linear (WL) filters have the capability to perform single antenna interference cancellation (SAIC) of one rectilinear or quasi-rectilinear (QR) co-channel interference (CCI). The SAIC technology for QR signals is operational in GSM handsets but requires enhancements for both VAMOS standard, an evolution of GSM/EDGE standard, and FBMC-OQAM networks, which are candidate for $5 \mathrm{G}$ mobile networks. In this context, we propose and analyze in this paper, for QR signals, a SAIC/MAIC enhancement based on the concept of three inputs WL FRESH filtering, exploiting almost exhaustively both the non-circularity and the cyclostationnarity of QR signals, contrary to classical approaches which only exploit very partly these properties.
\end{abstract}

Index Terms - Widely linear, SAIC, Quasi-rectilinear, Non-circular, CCI, Continuous-Time, Pseudo ML, FRESH

\section{INTRODUCTION}

Since two decades and the pioneer works on the subject [1-4], WL filtering has raised up a great interest for second-order (SO) non-circular signals [5] in many areas. Nevertheless, the application which has received the greatest interest is CCI mitigation in radio communication networks using rectilinear or QR modulations. Rectilinear modulations correspond to mono-dimensional modulations such as ASK or BPSK modulations. QR modulations are complex modulations corresponding, after a simple derotation operation [6], to a complex filtering of a rectilinear modulation. Examples of QR modulations are MSK, GMSK or OQAM modulations. One of the most important properties of WL filtering is its capability to perform SAIC of one rectilinear or QR multi-user (MU) CCI, allowing the separation of two users from only one receive antenna [7-9]. The powerfulness of this concept jointly with its low complexity explain why it is operational in most of GSM handsets, allowing significant network's capacity gains for the GSM system [9-10]. Extension of the SAIC concept to a multi-antenna reception is called MAIC. To further increase the spectral efficiency of speech services in emerging markets such as China or India, the Voice ser- vices over Adaptive Multi-user channels on One Slot (VAMOS) technology, has been recently standardized [11]. It enables the transmission of two GSM voice streams on the same TDMA slot at the same frequency through the Orthogonal Sub Channel (OSC) multiple access technique which aims at doubling the number of users served by a cell. The separation, at the handset level, of the two streams, potentially corrupted by co-channel OSC and/or GMSK interference, requires the implementation of enhanced SAIC techniques for QR signals, preliminary introduced in [12-14]. A similar need is also required to mitigate both inter-carrier interference (ICI) and CCI for networks which will use filter bank multi-carrier (FBMC) waveforms coupled with OQAM modulation, which are considered as promising candidates for the $5 \mathrm{G}$ mobile networks in particular [15]. First WL filtering based solutions are presented in [16-18].

In this context, the purpose of this paper is to propose an enhanced generic SAIC/MAIC technique for links using QR modulations, corrupted by QR CCI. Most of the available WL receivers [6-8], [12-14], [16-21], optimized for QR signals, implement a Time Invariant (TI) WL filter on the extended derotated observation vector and only exploit partly the noncircularity and the cyclostationarity properties of QR signals. To much better exploit these properties, we propose to use a particular Time Variant (TV) WL filter on the same extended observations, corresponding to a three inputs WL FREquency SHifted (FRESH) filter [2]. To show the powerfulness of this new philosophy, we adopt a continuous-time (CT) approach, allowing us to remove both the filtering structure constraints imposed by a discrete-time (DT) approach and the potential influence of the sample rate. Besides, we choose a pseudo Maximum Likelihood Sequence Estimation (MLSE) approach much more easy to compute than a MLSE approach. Note that the scarce papers dealing with WL FRESH filtering for demodulation of QR signals correspond to [22-24]. While [22] concerns DS-CDMA systems, [24] considers a particular DT MMSE approach. The concept of three inputs WL FRESH filter is only cited in [23] for interference cancellation in the GSM context but without any analysis and through a DT approach at the symbol rate, which finally reduces to the conventional WL approach. 


\section{MODELS AND SO STATISTICS}

\subsection{Observation model and SO statistics}

We consider an array of $N$ narrow-band antennas receiving the contribution of a QR signal of interest (SOI) and a total noise. The vector of complex amplitudes of the signals at the output of these antennas can be written as

$$
\mathbf{x}(t)=\sum_{k} j^{k} b_{k} \mathbf{g}(t-k T)+\mathbf{n}(t)
$$

Here, $b_{k}$ are real-valued zero-mean i.i.d. r.v., directly related to the SOI symbols [19] [8], $T$ is the symbol period for MSK and GMSK signals and half the symbol period for OQAM signals, $\mathbf{g}(t)=v(t) * \mathbf{h}(t)$ is the impulse response of the SOI global channel, * is the convolution operation, $v(t)$ and $\mathbf{h}(t)$ are the impulse responses of the SOI pulse shaping filter and propagation channel respectively and $\mathbf{n}(\mathrm{t})$ is the zero-mean total noise vector.

The SO statistics of $\mathbf{x}(t)$ are characterized by the two correlation matrices $\mathbf{R}_{x}(t, \tau)$ and $\mathbf{C}_{x}(t, \tau)$, defined by

$$
\begin{aligned}
& \mathbf{R}_{x}(t, \tau) \triangleq \mathrm{E}\left[\mathbf{x}(t+\tau / 2) \mathbf{x}^{H}(t-\tau / 2)\right] \\
& \mathbf{C}_{x}(t, \tau) \triangleq \mathrm{E}\left[\mathbf{x}(t+\tau / 2) \mathbf{x}^{T}(t-\tau / 2)\right]
\end{aligned}
$$

where ${ }^{T}$ and ${ }^{H}$ mean transpose and conjugate transpose respectively. Assuming that $\mathbf{n}(t)$ is composed of QR MU CCI and stationary background noise, it is easy to verify that $\mathbf{R}_{x}(t, \tau)$ and $\mathbf{C}_{x}(t, \tau)$ are periodic functions of $t$ with periods equal to $T$ and $2 T$, respectively. Matrices $\mathbf{R}_{x}(t, \tau)$ and $\mathbf{C}_{x}(t, \tau)$ have then Fourier series expansions given by

$$
\begin{aligned}
\mathbf{R}_{x}(t, \tau) & =\sum_{\alpha_{i}} \mathbf{R}_{x}^{\alpha_{i}}(\tau) e^{j 2 \pi \alpha_{i} t} \\
\mathbf{C}_{x}(t, \tau) & =\sum_{\beta_{i}} \mathbf{C}_{x}^{\beta_{i}}(\tau) e^{j 2 \pi \beta_{i} t} .
\end{aligned}
$$

Here, $\alpha_{i}$ and $\beta_{i}$ are the first and second SO cyclic frequencies of $\mathbf{x}(t)$, such that $\alpha_{i}=i / T$ and $\beta_{i}=(2 i+1) / 2 T, i \in \mathbb{Z}$ [25] [26], $\mathbf{R}_{x}^{\alpha_{i}}(\tau)$ and $\mathbf{C}_{x}^{\beta_{i}}(\tau)$ are the first and second cyclic correlation matrices of $\mathbf{x}(t)$ for the cyclic frequencies $\alpha_{i}$ and $\beta_{i}$ and the delay $\tau$, defined by

$$
\begin{aligned}
\mathbf{R}_{x}^{\alpha_{i}}(\tau) \triangleq & <\mathbf{R}_{x}(t, \tau) e^{-j 2 \pi \alpha_{i} t}> \\
\mathbf{C}_{x}^{\beta_{i}}(\tau) \triangleq & <\mathbf{C}_{x}(t, \tau) e^{-j 2 \pi \beta_{i} t}>
\end{aligned}
$$

where $<.>$ is the temporal mean operation in $t$ over an infinite observation duration.

\subsection{Extended derotated or two inputs FRESH model}

Conventional linear and WL processing of $\mathbf{x}(t)$ only exploit the information contained in the first $(\alpha=0)$ and first and second $(\alpha, \beta)=(0,0)$ zero SO cyclic frequencies of $\mathbf{x}(t)$ respectively. As no information is contained in $\beta=0$ for $\mathrm{QR}$ signals, a derotation preprocessing is required before $\mathrm{WL}$ filtering of QR signals. Using (1), the derotated observation vector can be written as

$$
\mathbf{x}_{d}(t) \triangleq j^{-t / T} \mathbf{x}(t)=\sum_{k} b_{k} \mathbf{g}_{d}(t-k T)+\mathbf{n}_{d}(t)
$$

where $\mathbf{g}_{d}(t) \triangleq j^{-t / T} \mathbf{g}(t)$ and $\mathbf{n}_{d}(t) \triangleq j^{-t / T} \mathbf{n}(t)$. Expression (8) shows that the derotation operation makes a QR signal looks like a rectilinear signal, with a non-zero information for $\beta=0$. Indeed, it is easy to verify that the two correlation matrices, $\mathbf{R}_{x_{d}}(t, \tau)$ and $\mathbf{C}_{x_{d}}(t, \tau)$ of $\mathbf{x}_{d}(t)$ are such that

$$
\begin{aligned}
& \mathbf{R}_{x_{d}}(t, \tau)=j^{-\tau / T} \mathbf{R}_{x}(t, \tau) \\
& \mathbf{C}_{x_{d}}(t, \tau)=j^{-2 t / T} \mathbf{C}_{x}(t, \tau) \triangleq e^{-j 2 \pi t / 2 T} \mathbf{C}_{x}(t, \tau)
\end{aligned}
$$

These expressions show that the first, $\alpha_{d_{i}}$, and second, $\beta_{d_{i}}$, SO cyclic frequencies of $\mathbf{x}_{d}(t)$ are such that $\alpha_{d_{i}}=\alpha_{i}$, and second, $\beta_{d_{i}}=\beta_{i}-1 / 2 T=i / T$, which proves the presence of information at $\beta_{d_{0}}=0$. Thus conventional WL processing of QR signals exploits the information contained in $\left(\alpha_{d_{0}}, \beta_{d_{0}}\right)=(0,0)$ through the exploitation of the temporal mean of the first correlation matrix of the extended derotated model

$$
\widetilde{\mathbf{x}}_{d}(t) \triangleq\left[\mathbf{x}_{d}^{T}(t), \mathbf{x}_{d}^{H}(t)\right]^{T}=\sum_{k} b_{k} \widetilde{\mathbf{g}}_{d}(t-k T)+\widetilde{\mathbf{n}}_{d}(t)
$$

where $\widetilde{\mathbf{g}}_{d}(t) \triangleq\left[\mathbf{g}_{d}^{T}(t), \mathbf{g}_{d}^{H}(t)\right]^{T}$ and $\widetilde{\mathbf{n}}_{d}(t) \triangleq\left[\mathbf{n}_{d}^{T}(t), \mathbf{n}_{d}^{H}(t)\right]^{T}$. Note that $\widetilde{\mathbf{x}}_{d}(t) \triangleq j^{-t / T} \mathbf{x}_{F_{2}}(t)$, where $\mathbf{x}_{F_{2}}(t) \triangleq\left[\mathbf{x}^{T}(t)\right.$, $\left.e^{j 2 \pi t / 2 T} \mathbf{x}^{H}(t)\right]^{T}$ is a particular two inputs FRESH model of $\mathbf{x}(t)$ which can be written as

$$
\mathbf{x}_{F_{2}}(t)=\sum_{k} j^{k} b_{k} \mathbf{g}_{F_{2}}(t-k T)+\mathbf{n}_{F_{2}}(t)
$$

where $\mathbf{n}_{F_{2}}(t)$ corresponds to $\mathbf{x}_{F_{2}}(t)$ with $\mathbf{n}(t)$ instead of $\mathbf{x}(t)$ and $\mathbf{g}_{F_{2}}(t) \triangleq\left[\mathbf{g}^{T}(t), e^{j 2 \pi t / 2 T} \mathbf{g}^{H}(t)\right]^{T}$ As the temporal mean of the first correlation matrices of $\widetilde{\mathbf{x}}_{d}(t)$ and $\mathbf{x}_{F_{2}}(t)$ contain the same information, TI linear processing of $\widetilde{\mathbf{x}}_{d}(t)$ and $\mathbf{x}_{F_{2}}(t)$ involving only this first correlation matrix are equivalent.

\subsection{Three inputs FRESH model}

While for rectilinear signals, the main information about their non-circularity is contained in $\beta=0$, for $\mathrm{QR}$ signals, it is symmetrically contained in $\left(\beta_{0}, \beta_{-1}\right)=(1 / 2 T,-1 / 2 T)$, or equivalently in $\left(\beta_{d_{0}}, \beta_{d_{-1}}\right)=(0,-1 / T)$. As models $\widetilde{\mathbf{x}}_{d}(t)$ or $\mathbf{x}_{F_{2}}(t)$ only exploit the information contained in $\left(\alpha_{d_{0}}, \beta_{d_{0}}\right)=(0,0)$, or $\left(\alpha_{0}, \beta_{0}\right)=(0,1 / 2 T)$, i.e. a part of the non-circularity information, they become sub-optimal. To overcome this limitation, we propose to exploit a three inputs FRESH model corresponding to

$$
\begin{aligned}
\mathbf{x}_{F_{3}}(t) & \triangleq\left[\mathbf{x}^{T}(t), e^{j 2 \pi t / 2 T} \mathbf{x}^{H}(t), e^{-j 2 \pi t / 2 T} \mathbf{x}^{H}(t)\right]^{T} \\
& =j^{t / T}\left[\widetilde{\mathbf{x}}_{d}^{T}(t), e^{-j 3 \pi t / 2 T} \mathbf{x}_{d}^{H}(t)\right]^{T} \triangleq j^{t / T} \mathbf{x}_{d_{F_{3}}}(t) \\
& =\sum_{k} j^{k} b_{k} \mathbf{g}_{F_{3}}(t-k T)+\mathbf{n}_{F_{3}}(t)
\end{aligned}
$$


where $\mathbf{n}_{F_{3}}(t)$ corresponds to $\mathbf{x}_{F_{3}}(t)$ with $\mathbf{n}(t)$ instead of $\mathbf{x}(t)$ and $\mathbf{g}_{F_{3}}(t) \triangleq\left[\mathbf{g}^{T}(t), e^{j 2 \pi t / 2 T} \mathbf{g}^{H}(t), e^{-j 2 \pi t / 2 T} \mathbf{g}^{H}(t)\right]^{T}$. It is straightforward to verify that the temporal mean of the first correlation matrices of $\mathbf{x}_{F_{3}}(t)$ and $\mathbf{x}_{d_{F_{3}}}(t)$ exploits the information contained in $\left(\alpha_{0}, \alpha_{-1}, \alpha_{1}, \beta_{0}, \beta_{-1}\right)=$ $(0,-1 / T, 1 / T, 1 / 2 T,-1 / 2 T)$, which allows us to exploit almost exhaustively both the cyclostationarity and the noncircularity of QR signals. Note that TI linear processing of $\mathbf{x}_{F_{3}}(t)$ or $\mathbf{x}_{d_{F_{3}}}(t)$ becomes now a TV WL filtering of both $\mathbf{x}(t)$ and $\mathbf{x}_{d}(t)$, called here three inputs WL FRESH filtering of $\mathbf{x}(t)$ or $\mathbf{x}_{d}(t)$.

\section{GENERIC PSEUDO-MLSE RECEIVER}

\subsection{Pseudo-MLSE approach}

To only exploit the information contained in the SO statistics of the observations, the CT MLSE receiver for the detection of the symbols $b_{k}$, would assume a Gaussian total noise despite the fact that the CCI are QR. Note that the Gaussian assumption would be verified in practice for a high number of i.i.d. CCI. Moreover, to take into account the SO cyclostationarity and the SO non-circularity properties of the CCI, the total noise would be assumed to be cyclostationary and noncircular. However, under these assumptions, the CT MLSE receiver, which optimally exploits the CCI SO properties, is very challenging to derive and even probably impossible to implement. Such a MLSE receiver would optimally exploit the information contained in all the $\left(\alpha_{i}, \beta_{i}\right), i \in \mathbb{Z}$, through the probable implementation of an infinite number of TI filters acting on an infinite number of FRESH versions of $\mathbf{x}(t)$ and $\mathbf{x}^{*}(t)$, where * means conjugate.

In this context, knowing that the CT MLSE receiver from $\mathbf{x}(t)$ (or $\mathbf{x}_{d}(t)$ ) in Gaussian non-circular stationary total noise $\mathbf{n}(t)$ (or $\mathbf{n}_{d}(t)$ ) is also the CT MLSE receiver from $\widetilde{\mathbf{x}}(t)$ (or $\widetilde{\mathbf{x}}_{d}(t)$ ) in Gaussian circular stationary extended total noise $\widetilde{\mathbf{n}}(t)$ (or $\widetilde{\mathbf{n}}_{d}(t)$ ) [27], a similar approach may be chosen to approximate the CT MLSE receiver in cyclostationary non circular total noise. The idea is then to choose an arbitrary finite number $M$ of FRESH versions of $\mathbf{x}(t)$ and/or $\mathbf{x}^{*}(t)$, generating the $M$ inputs FRESH model $\mathbf{x}_{F_{M}}(t)$, and to compute the CT MLSE receiver from $\mathbf{x}_{F_{M}}(t)$ assuming a circular, stationary and Gaussian $M$ inputs FRESH total noise vector $\mathbf{n}_{F_{M}}(t)$. This approach gives rise to the $M$ inputs pseudo-MLSE receiver associated with $\mathbf{x}_{F_{M}}(t)$. The problem is then to choose the vector $\mathbf{x}_{F_{M}}(t)$ of minimum size which generates a pseudo-MLSE receiver whose performance well approximates that of the CT MLSE receiver. In the following, we consider three observation models corresponding to $\mathbf{x}(t)$ (the conventional one), also denoted by $\mathbf{x}_{F_{1}}(t)$, $\mathbf{x}_{F_{2}}(t)$ (equivalent to $\widetilde{\mathbf{x}}_{d}(t)$, the conventional extended one) and $\mathbf{x}_{F_{3}}(t)$ (the proposed one) defined by (1), (12) and (13) respectively and we compare the output performance of the associated pseudo-MLSE receivers.

\subsection{Generic pseudo-MLSE receiver}

For a given value of $M(M=1,2,3)$, assuming a stationary, circular and Gaussian $M$ inputs total noise $\mathbf{n}_{F_{M}}(t)$, it is shown in [28] that the sequence $\widehat{\mathbf{b}} \triangleq\left(\widehat{b}^{1}, \ldots, \widehat{b}^{K}\right)$ which maximizes its likelihood from $\mathbf{x}_{F_{M}}(t)$ is the one which minimizes the following criterion: ${ }^{1}$

$$
\int\left[\mathbf{x}_{F_{M}}(f)-\mathbf{s}_{F_{M}}(f)\right]^{H}\left[\mathbf{R}_{n_{F_{M}}}^{0}(f)\right]^{-1}\left[\mathbf{x}_{F_{M}}(f)-\mathbf{s}_{F_{M}}(f)\right] d f
$$

Considering only terms that depend on the symbols $b_{k}$, the minimization of (14) is equivalent to that of the metric:

$$
\Lambda(\mathbf{b})=\sum_{k=1}^{K} \sum_{k^{\prime}=1}^{K} b_{k} b_{k^{\prime}} r_{k, k^{\prime}}-2 \sum_{k=1}^{K} b_{k} z_{F_{M}}(k)
$$

where $z_{F_{M}}(k) \triangleq \operatorname{Re}\left[j^{-k} y_{F_{M}}(k)\right]$ with

$$
\begin{gathered}
y_{F_{M}}(k)=\int \mathbf{g}_{F_{M}}^{H}(f)\left[\mathbf{R}_{n_{F_{M}}}^{0}(f)\right]^{-1} \mathbf{x}_{F_{M}}(f) e^{j 2 \pi f k T} d f, \quad(16) \\
r_{k, k^{\prime}}=j^{k^{\prime}-k} \int \mathbf{g}_{F_{M}}^{H}(f)\left[\mathbf{R}_{n_{F_{M}}}^{0}(f)\right]^{-1} \mathbf{g}_{F_{M}}(f) e^{j 2 \pi f\left(k-k^{\prime}\right) T} d f
\end{gathered}
$$

\subsection{Interpretation of the pseudo-MLSE receiver}

We deduce from (16) that $y_{F_{M}}(k)$ is the sampled version, at time $t=k T$, of the output of the TI filter whose frequency response is

$$
\mathbf{w}_{F_{M}}^{H}(f) \triangleq\left(\left[\mathbf{R}_{n_{F_{M}}}^{0}(f)\right]^{-1} \mathbf{g}_{F_{M}}(f)\right)^{H}
$$

and whose input is $x_{F_{M}}(t)$. The structure of the $M$ inputs pseudo-MLSE receiver is then depicted at Fig.1. It is composed of the TI $M$ inputs filter (18), followed by a sampling at the symbol rate, a derotation operation, a real part capture and a decision box implementing a modified version of the Viterbi algorithm.

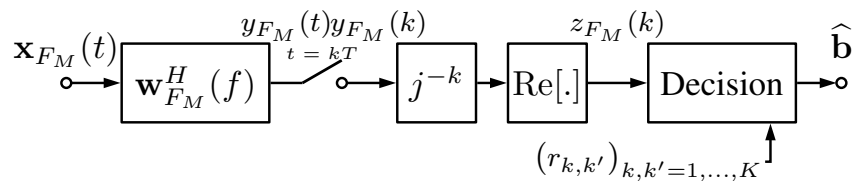

Fig.1 Structure of the $M$ inputs pseudo-MLSE receiver.

\subsection{SINR at the output of the pseudo-MLSE receiver}

For real-valued symbols $b_{k}$, the symbol error rate (SER) at the output of the $M$ inputs pseudo-MLSE receiver is directly linked to the signal to interference plus noise ratio (SINR) on the current symbol before decision, i.e., at the output $z_{F_{M}}(n)$ [29, Sec.10.1.4], while the inter-symbol interference is processed by the decision box. For this reason, we compute the expression of the output SINR hereafter and we will analyze

\footnotetext{
${ }^{1}$ All Fourier transforms of vectors $\mathbf{x}$ and matrices $\mathbf{X}$ use the same notation where $t$ and $\tau$ is simply replaced by $f$, e.g., here $\mathbf{R}_{n_{F_{M}}}^{0}(f)$ is the Fourier transform of (6), where $\alpha_{i}$ and $\mathbf{x}(t)$ are replaced by 0 and $\mathbf{n}_{F_{M}}(t)$ respectively, whereas $\mathbf{s}_{F_{M}}(f) \triangleq \sum_{k=1}^{K} j^{k} b_{k} \mathbf{g}_{F_{M}}(f) e^{-j 2 \pi f k T}$.
} 
its variations in particular situations in section 4. As $\mathbf{n}_{F_{M}}(t)$ is cyclostationary and non-circular, the filter (18) does not maximizes the output SINR and can only be considered as a $M$ inputs pseudo matched filter. It is easy to verify from (1), (12), (13), (16) and (17), that $z_{F_{M}}(n)$ can be written as

$$
z_{F_{M}}(n)=b_{n} r_{n, n}+\sum_{k \neq n} b_{k} \operatorname{Re}\left[r_{n, k}\right]+z_{n, F_{M}}(n),
$$

where $z_{n, F_{M}}(n) \triangleq \operatorname{Re}\left[j^{-n} y_{n, F_{M}}(n)\right]$ and $y_{n, F_{M}}(n)$ is defined by (16) for $k=n$ with $\mathbf{n}_{F_{M}}(n)$ instead of $\mathbf{x}_{F_{M}}(n)$. The SINR on the current symbol is then defined by

$$
\mathrm{SINR}_{\mathrm{M}} \triangleq \pi_{b} r_{n, n}^{2} / \mathrm{E}\left[\left(\operatorname{Re}\left[j^{-n} y_{n, F_{M}}(n)\right]\right)^{2}\right] \text {, }
$$

where $\pi_{b} \triangleq \mathrm{E}\left(b_{n}^{2}\right)$.

\section{SINR ANALYSIS FOR ONE CCI}

\subsection{Total noise model}

To show the powerfulness of (13) with respect to (11) or (12), we assume that the total noise is composed of one MU CCI and a background noise. Under these assumptions, $\mathbf{n}(t)$ can be written as

$$
\mathbf{n}(t)=\sum_{k} j^{k} e_{k} \mathbf{g}_{I}(t-k T)+\mathbf{u}(t)
$$

where $e_{k}$ are real-valued zero-mean i.i.d. r.v., directly related to the transmitted symbols of the MU CCI interference, $\mathbf{g}_{I}(t)=v(t) * \mathbf{h}_{I}(t), \mathbf{h}_{I}(t)$ is the impulse response of the propagation channel of the CCI and $\mathbf{u}(\mathrm{t})$ is the background noise vector, assumed stationary, temporally and spatially white. To simplify the following analysis, we assume a raised cosine pulse shaping filter $v(t)$ with a roll-off $\gamma$ and deterministic propagation channels with no delay spread such that

$$
\mathbf{h}(t)=\mu \delta(t) \mathbf{h} \quad \text { and } \quad \mathbf{h}_{I}(t)=\mu_{I} \delta\left(t-\tau_{I}\right) \mathbf{h}_{I}
$$

Here, $\mu$ and $\mu_{I}$ control the amplitude of the SOI and CCI, $\delta(t)$ is the Dirac pulse, $\tau_{I}$ is the delay of the CCI with respect to the SOI whereas $\mathbf{h}$ and $\mathbf{h}_{I}$, such that $\mathbf{h}^{H} \mathbf{h}=\mathbf{h}_{I}^{H} \mathbf{h}_{I}=N$, are the channel vectors of the SOI and CCI.

\subsection{SINR computations and analysis}

Under the previous assumptions, analytical interpretable expressions of the SINRs (20) are only possible for a zero rolloff. In this case, we denote by $\pi_{s} \triangleq \mu^{2} \pi_{b}, \pi_{I} \triangleq \mu_{I}^{2} \pi_{e}$ and $\eta_{2}$ the power of the SOI, the CCI and the background noise per antenna at the output of the pulse shaping matched filter, $\pi_{e} \triangleq \mathrm{E}\left[e_{n}^{2}\right], \varepsilon_{s} \triangleq \pi_{s} \mathbf{h}^{H} \mathbf{h} / \eta_{2}$ and $\varepsilon_{I} \triangleq \pi_{I} \mathbf{h}_{I}^{H} \mathbf{h}_{I} / \eta_{2}$. Moreover, assuming $N=1$ and a strong CCI $\left(\varepsilon_{I} \gg 1\right)$ for models (12) and (13), we obtain after tedious computations

$$
\begin{aligned}
\mathrm{SINR}_{1} & =\frac{2 \varepsilon_{s}}{1+\varepsilon_{I}\left[1-\cos \left(\frac{\pi \tau_{I}}{T}\right)+2 \cos \left(\frac{\pi \tau_{I}}{T}\right) \cos ^{2}\left(\phi_{I s}\right)\right]} \\
\mathrm{SINR}_{2} & \approx 2 \varepsilon_{s}\left[1-\frac{1+\cos ^{2}\left(\phi_{I s}+\frac{\pi \tau_{I}}{2 T}\right)}{2}\right] ; \Psi_{I s} \neq k \pi
\end{aligned}
$$

$$
\begin{gathered}
\mathrm{SINR}_{2} \approx \frac{\varepsilon_{s}}{\varepsilon_{I}} \frac{9}{2\left[3+2 \cos \left(4 \phi_{I s}\right)\right]} ; \quad \Psi_{I s}=k \pi \\
\mathrm{SINR}_{3} \approx 2 \varepsilon_{s}\left\{1-\frac{\left[\cos ^{2}\left(\phi_{I s}+\frac{\pi \tau_{I}}{2 T}\right)+\cos ^{2}\left(\phi_{I s}-\frac{\pi \tau_{I}}{2 T}\right)\right]}{2}\right\} \\
\left(\Psi_{I s}, \zeta_{I s}\right) \neq(k \pi, k \pi) \\
\mathrm{SINR}_{3} \approx \frac{\varepsilon_{s}}{\varepsilon_{I}} ; \quad\left(\Psi_{I s}, \zeta_{I s}\right)=(k \pi, k \pi)
\end{gathered}
$$

where $\phi_{I s} \triangleq \operatorname{Arg}\left(\mathbf{h}_{I}^{H} \mathbf{h}\right)$ is the phase difference between the SOI and the CCI, $\Psi_{I s} \triangleq \phi_{I s}+\pi \tau_{I} / 2 T$ and $\zeta_{I s} \triangleq \phi_{I s}-$ $\pi \tau_{I} / 2 T$. A receiver performs SAIC as $\varepsilon_{I} \rightarrow \infty$, if the associated SINR does not converge toward zero. We deduce from (23) that the conventional receiver performs SAIC very scarcely, only when $\left(\tau_{I} / T, \phi_{I s}\right)=\left(2 k_{1},\left(2 k_{2}-1\right) \pi / 2\right)$ or $\left(2 k_{1}+1, k_{2} \pi\right)$, where $k_{1}$ and $k_{2}$ are integer. However (24) and (26) show that the two and three inputs WL FRESH receivers perform SAIC as long as $\Psi_{I s} \neq k \pi$ and $\left(\Psi_{I s}, \zeta_{I s}\right) \neq$ $(k \pi, k \pi)$ respectively and are in this case such that $\mathrm{SINR}_{3} \geq$ $\mathrm{SINR}_{2}$, enlightening the great interest of (13). To give a statistical perspective of these results for arbitrary values of $\gamma$, we now assume that $\phi_{I s}$ and $\pi \tau_{I} / 2 T$ are independent r.v. uniformly distributed on $[0 ; 2 \pi]$. Under these assumptions, choosing $\varepsilon_{s}=10 \mathrm{~dB}$ and $\varepsilon_{I}=20 \mathrm{~dB}$, Fig.2 shows, for $M=1,2,3$ and $\gamma=0,0.5,1, \operatorname{Pr}\left[\left(\mathrm{SINR}_{\mathrm{M}} / 2 \varepsilon_{s}\right)_{d B} \geq x\right.$ $\mathrm{dB}] \triangleq p_{M}(x)$ as a function of $x(\mathrm{~dB})$. Note increasing performance with $\gamma$ for $M=2,3$ and the best performance of (13) with respect to (12) whatever $\gamma$. Note in particular, for $\gamma=0.5$ and $x=-3 \mathrm{~dB}$, that $p_{1}(x)=0 \%, p_{2}(x) \approx 26 \%$ and $p_{3}(x) \approx 63 \%$, proving the much better performance of (13) with respect to (12).

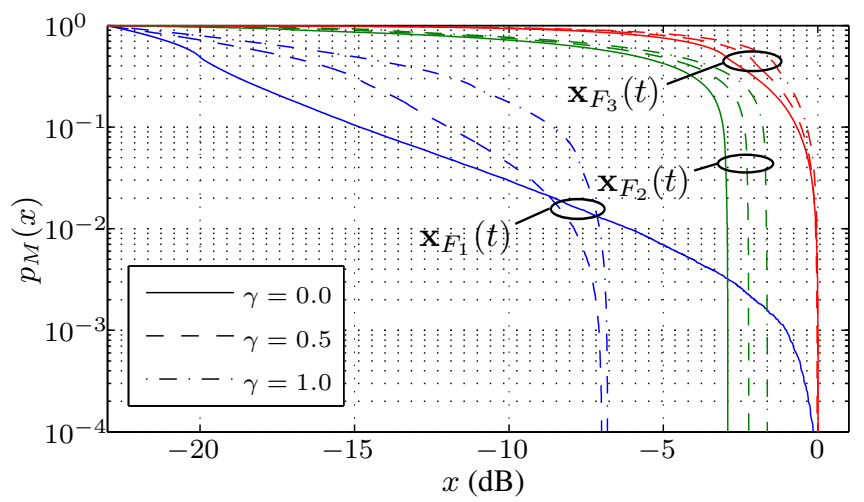

Fig.2 $p_{M}(x)$ as a function of $x, N=1, \varepsilon_{s}=10 \mathrm{~dB}, \varepsilon_{I}=20 \mathrm{~dB}$.

\section{CONCLUSION}

A SAIC/MAIC enhancement based on the concept of three inputs WL FRESH filtering has been proposed and analyzed in this paper, through a CT pseudo-MLSE approach, for MU CCI mitigation in networks using QR signals. This new approach has been shown to be much more powerful than the conventional WL approach, opening new perspectives for CCI mitigation in QR networks. Other approaches (DT, MMSE..) will be considered elsewhere. 


\section{REFERENCES}

[1] W.M. Brown and R.B. Crane, "Conjugate linear filtering", IEEE Trans. Inf. Theory, vol. 15, no. 4, pp. 462-465, July. 1969.

[2] W.A. Gardner, "Cyclic Wiener filtering: theory and method", IEEE Trans. Commun., vol. 41, no. 1, pp. 151-163, Jan. 1993.

[3] B. Picinbono and P. Chevalier, "Widely linear estimation with complex data", IEEE Trans. Signal Process., vol. 43, no. 8, pp. 2030-2033, Aug. 1995.

[4] P. Chevalier, "Optimal array processing for non stationary signals", Proc. ICASSP, pp. 2868-2871, Atlanta (USA), May 1996.

[5] B. Picinbono, "On Circularity”, IEEE Trans. Signal Process., vol. 42, no. 12, pp. 3473-3482, Dec 1994.

[6] Z. Ding and G. Li, "Single-Channel blind equalization for GSM cellular systems", IEEE J. Sel. Areas Communi., vol. 16, no. 8, pp. 1493-1505, Oct. 1998.

[7] H. Trigui and D.T.M. Slock, "Performance bounds for cochannel interference cancellation within the current GSM standard", Signal Processing, Elsevier, vol. 80, pp. 1335-1346, 2000.

[8] P. Chevalier and F. Pipon, ”New Insights into optimal widely linear array receivers for the demodulation of BPSK, MSK and GMSK signals corrupted by noncircular interferences - Application to SAIC", IEEE Trans. Signal Process., vol. 54, no. 3, pp. 870-883, March 2006.

[9] R. Meyer, W.H. Gerstacker, R. Schober, and J.B. Huber, "A single antenna interference cancellation algorithm for increased GSM capacity”, IEEE Trans. Wireless Commun., vol. 5, no. 7, pp. 1616-1621, July 2006.

[10] A. Mostafa, R. Kobylinski, I. Kostanic, and M. Austin, "Single Antenna Interference Cancellation (SAIC) for GSM networks", IEEE Proc. Vehicular Technology Conference (VTC04), vol 2, pp. 1089-1093, Oct. 2004.

[11] R.C.D. Paiva et al. "GSM voice evolution using Orthogonal Subchannels", IEEE Communications Magazine, pp. 80-86, Dec. 2012.

[12] M.G. Vutukuri, R. Malladi, K. Kuchi, and R.D. Koilpillai, "SAIC receiver algorithms for VAMOS downlink transmission", International Symposium on Wireless Communication Systems (ISWCS'11), pp. 31-35, Aug. 2011.

[13] D. Molteni and M. Nicoli, "Joint OSC receiver for evolved GSM/EDGE", IEEE Trans. Wireless Commun., vol. 12, no. 6, pp. 2608-2619, June 2013.

[14] M. Ruder, R. Meyer, F. Obernosterer, H. Kalveram, R. Schober, and W. Gerstacker, "Receiver concepts and resource allocation for OSC downlink transmission", IEEE Trans. Wireless Commun., vol. 13, no. 3, pp. 1568-1581, March 2014.

[15] G. Wunder et al. "5GNOW: Non-orthogonal asynchronous waveforms for future mobile applications", IEEE Commun. Magazine, pp. 97-105, Feb. 2014.

[16] M. Caus and A.I. Perez-Neira, "SINR analysis for a single interferenceMulti-stream transmission for highly frequencyselective channels in MIMO-FBMC/OQAM systems", IEEE Trans. Signal Process., vol. 62, no. 4, pp. 786-796, Feb. 2014.
[17] Y. Chen and M. Haardt, "Widely linear processing in MIMO FBMC/OQAM systems", International Symposium on Wireless Communication Systems (ISWCS'13), pp. 743-747, Aug. 2013.

[18] S. Josilo, M. Narandzic, S. Tomic, and S. Nedic, "Widely linear filtering based kindred CCI suppression in FBMC waveforms", International Symposium on Wireless Communication Systems (ISWCS'14), Barcelona, Aug. 2014.

[19] W.H. Gerstacker, R. Schober, and A. Lampe, "Receivers with widely linear processing for frequency-selective channels", IEEE Trans. Commun., vol. 51, no. 9, pp. 1512-1523, Sept. 2003.

[20] G. Gelli, L. Paura, and F. Verde, "On the existence of FIR zeroforcing equalizers for nonredundantly precoded transmissions through FIR channels", IEEE Signal Proc. Letters, vol. 12, no. 3, pp. 202-205, March 2005.

[21] D. Raphaeli, "A reduced complexity equalizer for OQPSK", IEEE Trans. Commun., vol 58, no. 1, pp. 46-51, Jan. 2010.

[22] A. Mirbagheri, K.N. Plataniotis, and S. Pasupathy, ”An enhanced Widely Linear CDMA receiver with OQPSK modulation”, IEEE Trans. Commun., vol. 54, no. 2, pp. 261-272, Feb. 2006.

[23] W.A. Gardner and C.W. Reed, "Making the most out of spectral redundancy in GSM: Cheap CCI suppression”, Proc. ASILOMAR Conf., pp. 883-889, Pacific-Greove, 2001.

[24] A.U.H. Sheikh and F. Hendessi, "FRESH-DFE: a new structure for interference cancellation", Wireless Personal Communications Journal, vol. 44, pp. 101-118, 2008.

[25] P. Gournay and P. Viravau, "Corrélation spectrale théorique des modulations CPM, Partie I: Résultat analytique pour les modulations CPFSK à 2 états (1-Rec)", Annals of Telecommunications, vol 53, no. 7-8, pp. 267-278, 1998.

[26] D. Vucic and M. Obradovic, "Spectral Correlation evaluation of MSK and Offset QPSK modulation", Signal Processing, vol. 78, pp. 363-367, 1999.

[27] S. Sallem, J.P. Delmas, and P. Chevalier, "Optimal SIMO MLSE receivers for the detection of linear modulation corrupted by noncircular interference," IEEE workshop on Statistical Signal Processing (SSP'2012), Ann Arbor, USA, Aug. 2012.

[28] G. Ungerboeck, "Adaptive maximum likelihood receiver for carrier-modulated data transmission systems", IEEE Trans. Commun., vol. 22, no. 5, pp. 624-636, May 1974.

[29] J.G. Proakis, Digital Communications, McGraw Hill Series in Electrical and Computer Engineering, 4th Edition, 2001. 